\title{
Hibridación relacional del Tercer Sector de Acción Social (TSAS) en la última década. Entre la movilización y las redes vecinales-comunitarias
}

\section{Sebastián Mora Rosado, Francisco Javier de Lorenzo Gilsanz}

RESUMEN: El tercer sector de acción social (en adelante, TSAS) es, desde el punto de vista teórico, un concepto elusivo y fluido, especialmente en el ámbito europeo. Los debates y las definiciones de consenso han sido numerosas para construir un concepto medible, compartido, compresivo y comparable. Estos esfuerzos han dado frutos notables en el desarrollo y análisis del TSAS. Ahora bien, el dinamismo de la realidad social en los últimos 10 años ha producido respuestas innovadoras y alternativas de la sociedad civil que han impactado en la cosmovisión compartida de los marcos conceptuales, las funciones y las acciones del TSAS. El TSAS se ha configurado como un espacio relacional híbrido que se desarrolla en tensión dinámica entre la movilización ciudadana, el emprendimiento social, la economía social y las redes comunitarias de apoyo. Esta tensión, que produce múltiples significados y perspectivas de análisis, solo es comprensible desde el análisis de las respuestas plurales que promueve la sociedad civil. El propósito de este artículo es analizar este proceso en el periodo de 10 años entre la Gran Recesión (2009-2012) y el Gran Confinamiento (2020) producido por el Covid-19. Debemos observar esta relacionalidad híbrida desde la intensificación de la presión asistencial que han supuesto ambas crisis para el conjunto de las organizaciones sociales. Esta tensión ha girado desde el dinamismo de los movimientos sociales en la Gran Recesión y el auge de las redes vecinales-comunitarias durante la pandemia.

PALABRAS CLAVE: TSAS, movilización ciudadana, emprendimiento social, economía social, redes comunitarias, redes vecinales, sociedad civil.

CLAVES ECONLIT: I31, I39, I38, I30. 
Cómo citar este artículo/How to cite this article: MORA, S. \& LORENZO, F.J. (2021): “Hibridación relacional del Tercer Sector de Acción Social en la última década. Entre la movilización y las redes vecinales-comunitarias", CIRIEC-España, Revista de Economía Pública, Social y Cooperativa, 103, 171-196. D0I: 10.7203/CIRIEC-E.103.21476.

Correspondencia: Sebastián Mora Rosado, Universidad Pontificia Comillas, ORCID: 00000001-9047-1989; Francisco Javier de Lorenzo Gilsanz, Cáritas española y Departamento de Trabajo social de UNED, ORCID: 0000-0001-6218-3584.

ABSTRACT: The third sector of social action (hereinafter TSAS) is, from a theoretical perspective, an elusive and fluid concept, especially in the European sphere. Debates and consensus definitions have been numerous to build a measurable, shared, comprehensive and comparable concept. These efforts have yielded remarkable results in the development and analysis of the TSAS. Now, the dynamism of social reality in the last 10 years has produced innovative and alternative responses from civil society that have impacted on the shared worldview of the conceptual frameworks, functions and actions of the TSAS.

The TSAS has been configured as a hybrid relational space that develops in dynamic tension between citizen mobilization, social entrepreneurship, the social economy and community support networks. This tension, which produces multiple meanings and perspectives for analysis, is only understandable from the analysis of the plural responses promoted by civil society.

The purpose of this article is to analyze this process in the 10-year period between the Great Recession (2009-2012) and the Great Stop (2020) produced by Covid-19.

We must understand this hybrid relationship from the intensification of the care pressure that both crises have meant for all social organizations. This tension has spun from the dynamism of social movements in the Great Recession and the rise of neighbourhood-community networks during the pandemic.

KEYWORDS: Citizen mobilization, social entrepreneurship, social economy community networks, neighbourhood networks, civil society. 


\section{Expanded abstract}

\section{Relational hybridization of Third Sector of Social Action in the last decade. Between mobilization and neighborhood-community networks}

The third sector of social action (hereinafter TSAS) is, from a theoretical perspective, an elusive and fluid concept, especially in the European sphere. Debates and consensus definitions have been numerous to build a measurable, shared, comprehensive and comparable concept. These efforts have yielded remarkable results in the development and analysis of the TSAS. Now, the dynamism of social reality in the last 10 years has produced innovative and alternative responses from civil society that have impacted on the shared worldview of the conceptual frameworks, functions and actions of the TSAS.

The TSAS has been configured as a hybrid relational space that develops in dynamic tension between citizen mobilization, social entrepreneurship, the social economy and community support networks. This tension, which produces multiple meanings and perspectives for analysis, is only understandable from the analysis of the plural responses promoted by civil society. The purpose of this article is to analyse this process in the 10 -year period between the Great Recession (2009-2012) and the Great Lockdown (2020) produced by Covid-19. We must understand this hybrid relationship from the intensification of the care pressure that both crises have meant for all social organizations. This tension has spun from the dynamism of social movements in the Great Recession and the rise of neighbourhood-community networks during the pandemic.

Throughout the following pages, we will discuss the impact of these neighborhood-community networks that achieved a high level of social legitimacy as a result of their efforts to de develop support and resilience building initiatives.

The topic will be addressed through a review of recent and specialized bibliography and seven interviews to people who hold different positions in the social field. These interviews have sought to reflect some territorial diversity: three have been carried out in cities with a population of 50,000 to 350,000 inhabitants and four in large cities with more than 500,000 inhabitants).

The role played by the TSAS during this period has not necessarily changed, but it has gained some new attributes. This is evolving to fit hybrid organizational forms characterised by open models that embrace diverse organizational cultures and flexible structures. In this process of hybridization, four essential intermediary fields can be differentiated: social economy, social entrepreneurship, social movements and new forms of community resilience. Strengthening 
these fields in a productive and innovative way will be a fundamental axis in the deployment of the TSAS in the coming years.

The TSAS in the Great Recession was developed in tension with emerging social mobilizations. These had a clear political profile compared to the TSAS care physiognomy. This profile has been shared among Southern European countries. However, during the Covid-19 crisis, the TSAS has maintained its care-oriented profile by sharing that space with the Community Neighborhood Networks created with a more social orientation. This type of community resilience, as shown by the first studies, seems to have been common in neighboring countries. In this sense, the TSAS lives with tension its lack of proximity and social base, as studies have shown for years in Spain.

This raises challenges that, depending on how they are addressed, may have an effect on their orientation, development and even on its organization:

- In relation to the care role, the TSAS needs to find alternative ways approaches that do not undermine the dignity of the people who request its help.

- In addition, the TSAS must develop its capacity to involve other agents (donors, companies, Public Administration...) in its evaluation processes and in relation to the search for solutions. This will entail "educational" processes in accordance with the orientations and objectives pursued by the TSAS (e.g., delivery of purse cards vs. delivery of food bags).

- The TSAS must develop its innovative capacity, which, unlike the public organisations (much more limited), will allow it to implement actions in different areas and in direct relationship with civil society and with neighborhood-community networks.

- But, in addition, it is necessary that the TSAS does not lose its political dimension (in a broad sense of the term) and that it delves into the roots and causes of the problems it addresses.

- This dimension affects its relationship with the Public Administration in two aspects, the first, in terms of political incidence (and even the complaint) directed at those who finance part of their activity; and the second, because the search for resources for this type of task usually requires a greater effort for the entities.

Finally, it should be noted that the TSAS daily presence in the various territories is a key element for the configuration of their relationships with other stakeholders. An important part of the recognition and potential alliances will depend on the continuity of this presence beyond periods of crisis. If one of the fundamental objectives of the TSAS is to promote the participation of those who are frequently excluded from various participation spheres (social, economic, political, cultural ...), their roots and links with the community (users, donors, volunteers, companies, Public Administration...) will be fundamental aspects for achieving this. For this, the TSAS should open up to engage with more open forms of governance, promoting the empowerment of affected people and communities and breaking the gap between professionals and participants. 


\section{Introducción}

El tercer sector de acción social (en adelante TSAS) es, desde el punto de vista teórico, un concepto elusivo y fluido (Salamon y Sokolowski, 2018), especialmente en el ámbito europeo. Los debates y las definiciones de consenso han sido numerosas para construir un concepto mensurable, compartido, compresivo y comparable ${ }^{1}$. Estos esfuerzos se alejan cada vez más de concepciones filosóficas, normativas e ideológicas intentando ofrecer perspectivas operativas, observables y susceptibles de ser verificadas en la práctica. Sin duda alguna, han dado frutos notables en el desarrollo y análisis del TSAS. Ahora bien, el dinamismo de la realidad social en los últimos 10 años, atravesada por dos profundas crisis, han producido respuestas innovadoras y alternativas de la sociedad civil que han impactado en la cosmovisión compartida de los contornos conceptuales, las funciones y los despliegues de acciones del TSAS.

El TSAS se ha configurado como un espacio relacional híbrido que se desarrolla en tensión dinámica con otros procesos y prácticas sociales. Así, el TSAS vive en tensión dinámica, como veremos, entre la movilización ciudadana, el emprendimiento social, la economía social y las redes comunitarias de apoyo. Esta tensión dinámica, que produce múltiples significados y perspectivas de análisis, solo es comprensible desde el análisis de las respuestas plurales que promueve la sociedad civil.

El propósito de este artículo es analizar este proceso de relación e hibridación que acaece en el TSAS en el periodo de 10 años entre la Gran Recesión (2009-2012) y el Gran Confinamiento (2020) producido por el Covid-19.

Esta relacionalidad híbrida, a modo de tipos ideales, debemos observarla en España desde la intensificación de la presión asistencial que han supuesto ambas crisis para el conjunto de las organizaciones sociales. Esta tensión ha girado desde el dinamismo de los movimientos sociales en la Gran Recesión y el auge de las redes vecinales-comunitarias durante la pandemia.

A lo largo de las siguientes páginas, se prestará especial atención al impacto de estas redes vecinales-comunitarias que han adquirido una gran legitimidad social desde las labores de apoyo y construcción de resiliencia cívica.

Existen trabajos relevantes que aportan un amplio material para el análisis de algunas de las cuestiones mencionadas. De entre ellos, cabe destacar el coordinado en 2015 por Rodríguez Cabrero y Marbán, el cual recoge un estudio Delphi a 31 personas expertas, y en el que se abordan cuestiones como la situación del TSAS, el impacto de la crisis de 2008, los retos en el medio y largo plazo y su relación con los movimientos sociales.

Junto a éste, otros trabajos (EAPN-ES, 2019; Chaves, R y Zimmer, 2017) también abordan algunos de estos aspectos. A pesar de ello, hemos considerado oportuna la realización de 7 entrevistas en profundidad, a personas que ocupan diversas posiciones en el ámbito social. Dichas entrevistas han buscado una cierta diversidad territorial: 3 en ciudades de entre $50.000 \mathrm{y}$ 350.000 habitantes y otras 4 en ciudades de tamaño grande de más de 500.000 habitantes). La

1. Un esfuerzo destacado se desarrolló con el programa Third Sector Impact del programa FP7 de la Unión Europea. Recuperado de https://cordis.europa.eu/project/id/613034/es 
aportación más significativa de estas entrevistas tiene relación con dos cuestiones concretas: la primera es la proximidad temporal al objeto de estudio, ya que ninguna de las fuentes mencionadas recoge las particularidades -en caso de darse -de lo ocurrido durante los primeros meses de pandemia; la segunda, es el acercamiento a una cuestión concreta que tampoco aparece de manera expresa en los materiales elaborados anteriormente y que mostramos como un factor determinante: el auge de las redes vecinales-comunitarias. Por lo tanto, la realización de estas entrevistas debe considerarse con un fin exploratorio, pues como señalan autores como Vallés (1999) o Batthyány y Cabrera (2011) este tipo de estudios generalmente sirven para examinar temas poco estudiados o que no ha sido abordado antes. Explorar los emergentes discursivos (Conde, 2019) en esta nueva situación social es un aspecto relevante para la aproximación al TSAS y el planteamiento de futuras investigaciones².

El papel desempeñado por el TSAS durante este periodo no necesariamente ha cambiado, pero sí se le ha atribuido (tanto interna y como externamente) algunas diferencias significativas. Especialmente, en esa relación comparativa con quienes ha compartido espacios dentro del (imaginario) eje de acción política/asistencial. Más allá de las tareas desarrolladas o de los proyectos concretos llevados a cabo, surgen otras cuestiones relevantes a la hora de analizar al TSAS: su relación con la Administración pública, la existencia o no de una base social implicada en cuestiones que vayan más allá de la financiación, su presencia en los territorios más afectados por las crisis... Cuestiones que, en definitiva, tienen consecuencias directas sobre su sentido e identidad.

\section{Dinamismo de hibridación organizativa e ideológica del TSAS}

El TSAS en Europa están evolucionando hacia formas organizativas híbridas que integran, bajo un mismo paraguas, realidades dotadas de diversas culturas e identidades institucionales. Si se considera el conjunto del tercer sector (en adelante TS), sus contornos aparecen cada vez más difusos. La hibridación cultural, organizativa e institucional es la fisonomía que va adquiriendo el TSAS en Europa (Pahl y Zimmer, 2017: 151-153). La creciente complejidad social y económica hacen evolucionar al TSAS hacía un modelo abierto que acoge culturas organizativas diversas y estructuras flexibles. Se desarrolla por sectores de actividad que traspasan las fronteras tradicionales del sector y, se visualiza claramente como el TSAS navega hacia una mixtura institucional que despliega diversas lógicas de acción en proyectos participados por ámbitos diversos. En este sentido el TSAS es como una estación intermodal (Fantova, 2019) o sistema intermediario entre escenarios diversos (Stanzani, 2005: 205).

2. Como más adelante señalamos, entre otros, la emergencia de la autoorganización y la autogestión, la inmediatez de la organización por las redes sociales digitales, la simetría de los participantes no aparecía de manera nítida en estudios anteriores. 
Podemos agrupar la hibridación relacional en el TSAS en cuatro campos intermediarios esenciales que han mostrado especial relevancia en los últimos 10 años (gráfico 1). A pesar de la solidez que muestra el TSAS en los diversos países europeos, este se ve sostenido e impulsado por tensiones institucionales, económicas, políticas, conceptuales y pragmáticas que le hacen estar en continuo desarrollo.

Estos campos intermediarios, que analizaremos a continuación, son: la economía social, el emprendimiento social, los movimientos sociales y las nuevas formas de resiliencia comunitaria.

\section{Gráfico 1. Campos intermediarios de hibridación del TSAS}

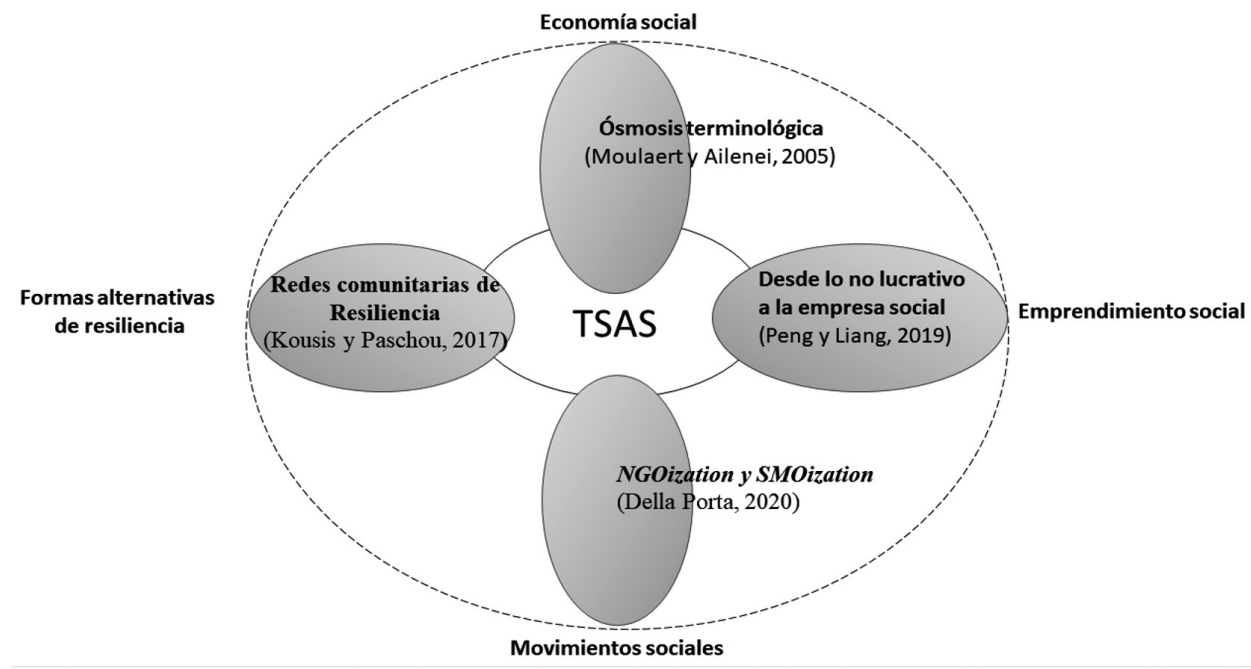

Fuente: Elaboración propia.

Un primer ámbito de hibridación del TSAS se construye desde la relación con la economía social y solidaria. Es sabido que el TSAS se ha construido en torno a dos tradiciones esenciales: la tradición continental -que hermana al TSAS con la economía social en sus diferentes variantes -y, desde otro punto de vista, la tradición anglosajona, que sitúa al TSAS en la órbita de lo no-lucrativo.

La tradición continental concibe la economía social como un tercer sector de las economías, situado entre el sector público y el privado tradicional (Monzón y Chaves, 2019). Esta economía social tiene dos subsectores dinamizando el espacio social: subsector de mercado y el subsector de no-mercado. El primero abarcaría la clásica visión de la economía social (cooperativas, mutuas, mutualidades, empresas sociales...) y, el segundo, el entramado de asociaciones denominado "TSAS, que suministran a las familias de forma gratuita bienes sociales o preferentes de indudable utilidad social" (Chaves, Monzón y Zaragoza, 2013:22). 
Esta consustancial relacionalidad, entre la economía social y el TSAS, se ve intensificada en periodos de crisis, produciendo una "ósmosis terminológica" en el ámbito de la economía social y el TSAS que solo son comprensibles a la luz de los contextos y las prácticas sociales (Moulaert y Ailenei, 2005).

En los últimos años se está produciendo una clara hibridación del TSAS con las diversas formas de emprendimiento social ${ }^{3}$. Estas formas innovadoras están siendo muy analizadas y consideradas como un factor de desarrollo necesario en el ámbito del TSAS (Maier, Meyer y Steinbereithner, 2016; Peng y Liang, 2019). Que las organizaciones del TSAS evolucionen hacia algún tipo de emprendimiento social y que uno de sus objetivos sea la promoción de emprendedores sociales ha calado de manera intensa. Algunos autores señalan el peligro de mercantilización del campo social que esta relacionalidad puede producir en el TSAS (Estivill, 2015:12) enmarcada en las tendencias "filantrocapitalistas" (Bishop y Green, 2009) que reflejan la alianza entre "los intereses financieros, las escuelas de negocios y el estado neoliberal orientada a cubrir la fractura material e ideológica dejada por la crisis económica" (Valenzuela-García, Molina, Lubbers, Escribano y Fuentes, 2019: 371).

Desde el plano de la movilización social el TSAS ha estado referido a los movimientos sociales en los últimos treinta años. Se ha producido, en formulación de Donatella Della Porta (2020), una NGOization of social movement organizations y la SMOization of civil society, generando unos espacios intersticiales que rompen con la lógica dicotómica ${ }^{4}$. Ya a finales de los años 90 en España, el TSAS fue bautizado como movimiento de solidaridad adquiriendo un protagonismo significativo.

Los movimientos de solidaridad se diferenciaban de los movimientos más tradicionales en tres aspectos fundamentales (Ibarra y Tejerina, 1998: 10 y ss): la práctica esencial es la solidaridad, los medios de acción colectiva para perseguir los fines son diferentes a los desplegados por los movimientos tradicionales y presentan una identidad colectiva más difusa con mayor estructura institucional.

Por último, es clave entender lo que sucedió en Europa a partir de la crisis en la Gran Recesión. El impacto intenso sobre las poblaciones más vulnerables generó un abanico plural de respuestas de la sociedad civil. Surgieron infinidad de iniciativas ciudadanas y colectivas para afrontar los tiempos económicos difíciles que fueron analizadas como Formas Alternativas de Resiliencia (en adelante FAR) (Kousis y Paschou, 2017) u Organizaciones de Acción Alternativa (Giudni y Grasso, 2018; Zamponi y Bosi, 2018). La pandemia del Covid-19 ha reactivado esta resiliencia comunitaria como un valor central, más allá de los entramados institucionales formales públicos y privados (Paarlberg, LePere-Schloop, Walk, Ai y Ming, 2020).

Estas respuestas, como veremos con más detenimiento, enfatizan la dimensión local, la autoorganización comunitaria y la participación de los afectados en las respuestas a las necesidades sufridas (Moulaert, Martinelli, Swyngedouw y González, 2005). Nosotros utilizaremos

3. Entendemos que el emprendimiento social pertenece a una cultura diversa que la economía social y persigue los objetivos de manera diferente, aunque tiene ámbitos y objetivos comunes con la economía social (Sanz, 2019). 4. El adjetivo intersticial se emplea en teoría social para describir distintos tipos de procesos que se dan en los espacios y grietas dentro de alguna estructura social dominante para recrear innovaciones sociales (Wright, 2014). 
el término redes vecinales-comunitarias (Ministerio de Sanidad, 2020), reconociendo que solo abarca una parte de las FAR, definidas como "las estructuras informales que han nacido espontáneamente en el seno de la sociedad civil. Estas redes conectan vecinas y vecinos, centros sanitarios, colegios, empresas, etc. para dar una respuesta coordinada a las necesidades de un área determinada surgidas a causa de la crisis de la Covid-19, permitiendo poner en marcha ágilmente soluciones efectivas y originales. La fuerza de las redes se basa en la agilidad, flexibilidad, rapidez y su cercanía a los problemas" (Buj y Caso, 2021: 6).

Por último, y a pesar de que no es objeto de estudio en este artículo, no podemos obviar el rol que el tercer sector de acción social desempeña también como agente o instrumento de las políticas públicas y, específicamente, de los sistemas públicos de servicios sociales. Las entidades del tercer sector de acción social en nuestro país son, en muchos casos, prestadoras de servicios contemplados en los catálogos de las comunidades autónomas o municipios, y son además financiados con fondos públicos. Las conexiones que se derivan de esta relación podrían ser identificadas también como parte de otro proceso de hibridación que afecta a la naturaleza e identidad de estas entidades.

\section{La presión asistencial como eje de interpretación}

Desde la Gran Recesión los países considerados de rentas altas se han visto impactados por un incremento intenso de las necesidades básicas de parte de su población. La tasa de personas en riesgo de pobreza (UE-15) llegó en 2012, según datos de Eurostat, al 23\%; y los países de Sur de Europa fueron especialmente impactados por la crisis.

En España los diversos Informes FOESSA $(2014 ; 2019)$ han puesto reiteradamente de manifiesto esta intensa fragilidad social. Este acrecentamiento de las necesidades primarias en la población conllevó en estos países una ampliación considerable, por ejemplo, de los bancos de alimentos expresión última de la severidad de la situación (Gentilini, 2013; Pérez de Armiño, 2014).

Estas prácticas dejaron de ser consideradas como una huella del pasado beneficiente para convertirse en un campo de innovación (Meijs, Handy, Simons y Roza, 2019). Surgieron en Europa un sinfín de respuestas públicas y privadas para tratar de paliar estas situaciones ${ }^{5}$ creando un campo de innovaciones sociales relevantes.

En este periodo, el TSAS en España desempeñó un rol de "asistencia" muy intenso (Rodríguez Cabrero y Marbán, 2015). Desde el año 2008 al 2013, periodo cumbre del impacto de la crisis, en el TSAS se incrementaron en más de 10 millones las atenciones a personas vulnera-

5. El programa "LIVEWHAT Project. LIVING WITH HARD TIMES" FP7 de la Unión Europea reflejó muchas de dichas iniciativas desde al campo de resiliencia comunitaria. Recuperado de https://www.unige.ch/livewhat/ 
bles (Gómez, 2020). Este impacto significó en estas entidades un cambio en la orientación de muchos de sus programas.

Tomando como ejemplo a Cruz Roja y Cáritas Española, como entidades singulares del TSAS, podemos observar esta evolución. Cruz Roja, en su programa de reparto de alimentos, llegó a ayudar entre los años 2012 al 2014 (Tabla 1) a más de un millón de personas por año. En el año 2017, con la recuperación económica, más de 700.000 personas quedaron en el programa.

\section{Tabla 1. Cruz Roja Programa de Reparto de alimentos}

\begin{tabular}{|c|c|c|}
\hline Año & \multicolumn{2}{|c|}{ Personas beneficiarias } \\
\hline 2012. & $\ldots$ & 1.030 .571 \\
\hline 2013. & & 1.065 .909 \\
\hline 2014. & & 1.103 .47 \\
\hline 2015. & ........ & 843.40 \\
\hline 2016. & ........ & 777.15 \\
\hline 2017. & $\ldots$ & 728.0 \\
\hline
\end{tabular}

Fuente: Elaboración propia a partir de las Memorias de Cruz Roja Española.

Gráfico 2. Personas atendidas en el Programa de Acogida y Asistencia de Cáritas Española

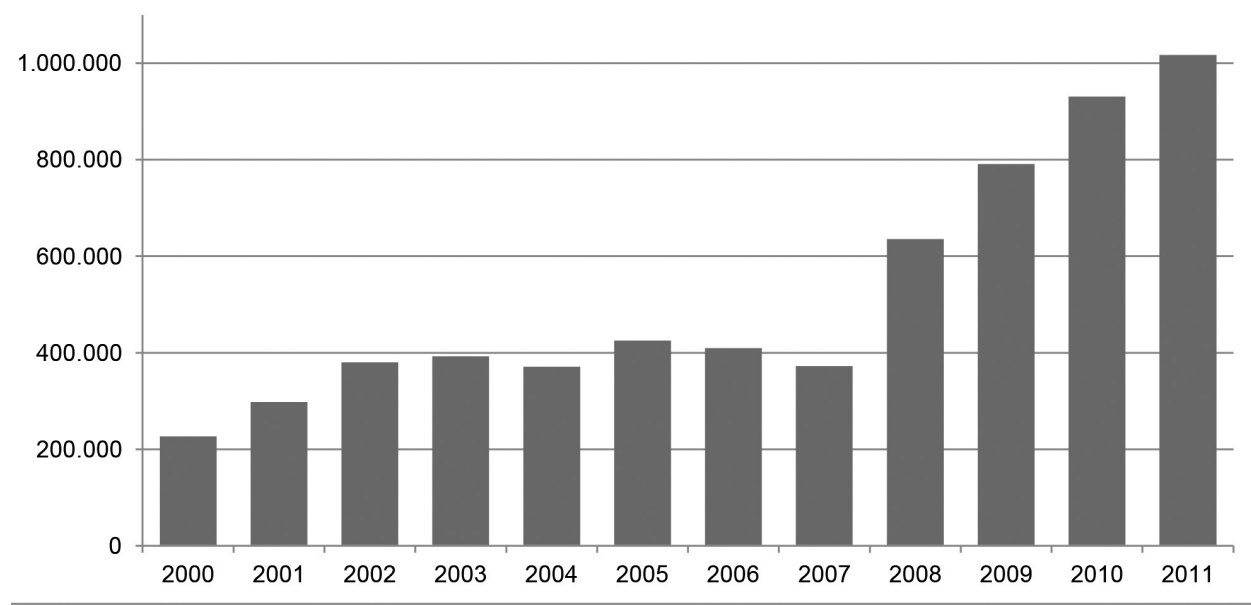

Fuente: VII Observatorio de la realidad social de Cáritas Española. 
También Cáritas (Gráfico 2) sufrió el impacto de la crisis en sus primeros años: duplicó, en cinco años, el número de personas acompañadas en el programa de Asistencia (en 2011 más de un millón de personas estaban siendo atendidas). En el año 2011 se inició un tenue descenso de las personas beneficiadas en los Programas de Acogida y Asistencia hasta 2015, año en el cual la bajada de personas fue considerable situándose, según la Memoria de ese año, en torno a 800.000 personas. Esto significaba, al igual que en Cruz Roja, niveles superiores al 2008 en el inicio de la crisis.

La situación social, las políticas de austeridad y el imaginario de la crisis produjeron también una clara asistencialización de las Políticas sociales ${ }^{6}$. Desde la práctica profesional, los directores de centros de servicios sociales denunciaban la asistencialización de los Servicios Sociales desde "la proliferación y diversidad de ayudas para paliar carencias materiales (vivienda, energía, alimentos...) convierten a los servicios sociales en oficinas de cheking social para clasificar a los ciudadanos" (García et al., 2017: 4). En expresión de García Roca (2012) los servicios sociales se pusieron en "pie de emergencia".

La pandemia producida por el coronavirus está suponiendo un nuevo impacto social de intensidad inusitada (Mora, 2020). A nivel europeo la inseguridad alimentaria se está incrementando, convirtiéndose en un campo de acción prioritario de las entidades sociales. Los datos que recoge la encuesta de Eurofound (2020) muestran, cómo al principio de la pandemia, un $30 \%$ de los hogares europeos tuvieron que hacer cambios en la dieta -ajustar la cesta de la compra -, mientras que menos del 5\% de los hogares, en la media UE-27 y en España en el mes de julio, recibieron alguna ayuda pública para cubrir gastos del hogar, bonificaciones, vales 0 comida. El porcentaje de hogares que había solicitado o recibido ayuda de alguna entidad del TSAS u otras organizaciones sociales (tabla 2) para la media UE-27 era del 1,8\% en el mes de julio y del 2,1\% en España.

\section{Tabla 2. Ayudas de organizaciones del TSAS y otras organizaciones (julio 2020)}

\begin{tabular}{l|c|c} 
& España & UE-27 \\
\hline Ha recibido & $2,1 \%$ & $1,8 \%$ \\
\hline Ha solicitado, pero no recibió aún & $1,2 \%$ & $0,7 \%$ \\
\hline Ha solicitado y se le ha denegado & $0,7 \%$ & $0,8 \%$ \\
\hline Total & $\mathbf{4 , 0} \%$ & $\mathbf{3 , 3} \%$ \\
\hline
\end{tabular}

Fuente: Elaboración propia. Eurofound 2020.

6. A modo de ejemplo, en el año 2013 el Ministerio en la convocatoria de programas con fines de interés social con cargo al 0,7\% de IRPF (en aquellos años era de carácter estatal) introduce por primera vez los programas de urgencia social con una dotación presupuestaria muy importante. 
En España, los informes de algunas organizaciones del TSAS (Cáritas, 2021; Cruz Roja, 2021) y la última Encuesta de Condiciones de Vida (2021) ponen de manifiesto el proceso de "empobrecimiento de la pobreza" o crecimiento de la pobreza en sus versiones más severas.

Las popularizadas "colas del hambre", más allá de lo que signifiquen realmente, han sido la imagen plástica de este incremento. Cruz Roja (2021) cifra en un 21,6\% el incremento de las necesidades básicas de las personas que atiende en sus programas. El crecimiento de los hogares sin ingresos en la población atendida por Cáritas en febrero de 2020 estaba en torno al 12\%, llegó a un máximo del 28\% en abril del 2020 para empezar en año 2021 en el 17\%, cinco puntos en menos de un año. Por su parte, el $22 \%$ de los hogares atendidos por Cruz Roja no tienen ningún tipo de ingreso, lo que supone un impacto de la Tasa AROPE, casi veinte puntos superiores, en las personas atendidas durante la pandemia. Ante esta realidad, Cruz Roja lanzó el Plan Responde impulsando la mayor operación de la organización en territorio español ${ }^{7}$. Cáritas por su parte, con el Plan Cáritas ante el coronavirus, cifraba en su última Memoria (2020) en 1.425.991, el 80,6\% del total, las personas atendidas en los programas de Acogida y asistencia (lo que supone 625.991 personas más que en 2015).

La Gran Recesión y el Gran Confinamiento producido por el coronavirus han supuesto un incremento importante de la pobreza en sus niveles más severos. Esto ha supuesto un reto para las sociedades, para las políticas públicas y para el TSAS. Este último ha incrementado desde el año 2008 de manera considerable su labor asistencial en el entorno europeo en general (Pape et al., 2017) y en el español en particular (Rodríguez Cabrero y Marbán, 2015). Esta labor asistencial se ha convertido en un eje de interpretación esencial para, a partir de él, observar las dinámicas de hibridación relacional y la emergencia de nuevos actores sociales en el campo de lo social.

\section{El dinamismo del TSAS en España: entre la movilización y las redes comunitarias}

El eje asistencial, como hemos analizado, ha influido de una manera decisiva en la orientación, el desarrollo y la organización del TSAS. Aunque con diferencias, tanto en la Gran Recesión, como durante la pandemia, la carga asistencial (prestación de servicios) ha constituido la fisonomía más nítida del sector.

7. Cruz Roja lanzó el Plan Responde en marzo del 2020 y lo catalogó como "la mayor movilización de recursos, capacidades y personas de Cruz Roja en nuestra historia, para brindar asistencia a personas en vulnerabilidad". https://www2.cruzroja.es/-/lanzamos-el-plan-cruz-roja-responde-frente-al-covid-19. 
No olvidemos que, por un lado, están las necesidades básicas concretas, que hay que atenderlas sí o sí, nos guste o no. Pero, además, a nivel social, la imagen más asistencial es la que triunfa.

(Entrevista 1)

En España el TSAS ha sufrido procesos relevantes de manera acelerada en estos últimos 10 años: la crisis económica y social del 2009-2014 con repercusiones en el ser y el quehacer del TSAS (POAS, 2015; Ruiz Villafranca, 2016); un proceso de "cohesión interna" que culminó con la creación de la Plataforma del Tercer Sector (Plataforma del Tercer Sector, 2011); la emergencia de una movilización social sin precedentes en torno al 15M y la compleja relación con el TSAS (Zubero, 2015); la promulgación de la Ley 43/2015, de 9 de octubre, del Tercer Sector de Acción Social; el impacto sin precedentes de la crisis social producida por el coronavirus (CÁRITAS, 2021; CRUZ R0JA, 2021) y la reactivación de las redes vecinales-comunitarias en labores de asistencia a las necesidades básicas de la ciudadanía (Ministerio de Sanidad, 2020; Vidal, 2021).

Si atendemos a los escenarios de crisis (Gran Recesión 2009-2014 y el Gran Confinamiento 2020-2021) podemos observar, a nivel español y europeo, la multiplicación de respuestas de la sociedad civil, en sus diversas dimensiones, para tratar de atender y paliar las consecuencias de las crisis. Es conocido que en tiempos de crisis económicas florecen iniciativas innovadoras y se recrean respuestas tradicionales para encarar los impactos sociales de las mismas (Kousis, 2017; Zamponi y Bosi, 2018). Esta afirmación es compatible, como algunos estudios muestran (Cameron, 2021), con el retraimiento del asociacionismo voluntario en instituciones formales en tiempos de crisis.

Sí, han surgido iniciativas, varias. Al principio más individuales, hacia la gente más cercana. Luego más amplias y organizadas. Es lo normal. La duda es si van a perdurar o si tan solo son respuestas de carácter emocional.

(Entrevista 7)

Moulaert y Aileni trazan el desarrollo histórico de la economía social, el TS y las economías solidarias desde las innovaciones sociales surgidas en las épocas de profundas crisis (2005).

Es el TS quien debe innovar. A la Administración le resulta muy difícil hacerlo. Debe ser el TS y la sociedad civil.

(Entrevista 6)

Estas innovaciones, desde la primera Revolución industrial a nuestros días, han ido apareciendo como "olas de solidaridad". Para Kousis y Paschou (2017) en la Gran Recesión apareció una "quinta ola de estructuras de solidaridad" conformada por iniciativas estratégicas creadas por los ciudadanos en la esfera pública, que ofrecían alternativas para soportar el día a día relacionadas principalmente con necesidades urgentes (vivienda, alimentación, salud, vestido, educación) e intentando transformar políticamente la realidad.

La crisis de 2008 comenzó poniendo sobre la mesa dos cuestiones básicas, vivienda y empleo, que rápidamente le dieron un carácter político.

(Entrevista 4) 
Estas redes vecinales-comunitarias de resiliencia son experiencias que surgen de una gobernanza abierta, con un dinamismo botton-up y una amplia base social (Moulaert et al., 2005). Promueven de forma explícita el empoderamiento de las personas y comunidades afectadas, otorgando una fuerte valoración al apoyo entre pares y promoviendo la ruptura del gap entre profesionales y participantes (Evers y Ewert, 2015). Además, tienden a ser iniciativas que politizan las acciones de solidaridad desde las experiencias locales (Giudni y Grasso, 2018; Zamponi y Bosi, 2018).

Se trabaja desde las capacidades de cada persona, desde lo que cada uno pueda aportar. No había estructura pensadas porque no se tenía previsto algo tan grande (...) Somos asamblearios y horizontales (...) Se empezó a crear el "camino a la autogestión" y no se montó para ayudar a personas pobres sino para ayudarse entre las vecinas.

(Entrevista 2)

El análisis que ha prevalecido sobre estas nuevas estructuras de solidaridad está enmarcado entre dos ejes básicos: el dinamismo de los movimientos sociales y la emergencia de la resiliencia comunitaria (Kousis, 2017). Aunque ambos ejes son complementarios, si enfatizamos el eje de los movimientos sociales las experiencias tienen un carácter más político y, sin embargo, si resaltamos la resiliencia comunitaria las iniciativas tienen una fisonomía más asistencial. El planteamiento que aportamos en nuestro análisis es que en España (Gráfico 3) estas respuestas innovadoras resaltaron el eje movilizador durante la Gran Recesión, y están destacando el eje de resiliencia comunitaria durante la crisis de coronavirus. Es decir, fueron más proclives a la politización durante la crisis financiera del 2009-2014 y la respuesta a la pandemia está siendo más dirigida a las acciones directas para ayudar a otros o a sí mismos en las necesidades básicas.

Han sido dos crisis diferentes. La primera cuestión que las distingue es el confinamiento absoluto, que ha afectado a los propios movimientos. En la del 2008 había una interacción con la gente que lo pasaba mal, y se encauzaba a las entidades formales; en esta crisis no tanto, porque los movimientos inicialmente no se planteaban la prestación; se planteaban el encauzamiento, pero estaban cerradas las estructuras formales. Otro elemento diferencial es la urgencia de las necesidades. En concreto lo relativo a las necesidades que se satisfacen a través de colegios (comedores)... y los colegios cerraron. En el 2008 tuvo más carga política, en sentido de denuncia, porque había un problema político. Ahora, lo político se ha trasladado a las iniciativas vecinales. Es también una dimensión política entendida desde la cercanía, no desde lo institucional.

(Entrevista 5)

El TSAS, en primer lugar, se orientó hacia lo laboral (formación, empleo...). Pero fue implicándose progresivamente en todas las cuestiones relacionadas. Y se evidenció entonces el rol de los bancos, los desahucios... cuestiones que la cobertura de necesidades materiales se percibiera como consecuencias de una cuestión política.

(Entrevista 4) 


\section{Gráfico 3. Ejes de las nuevas estructuras de solidaridad (redes comunitarias) en España}

\section{Gran Recesión (2009-2014) Gran Parón (2020-2021)}

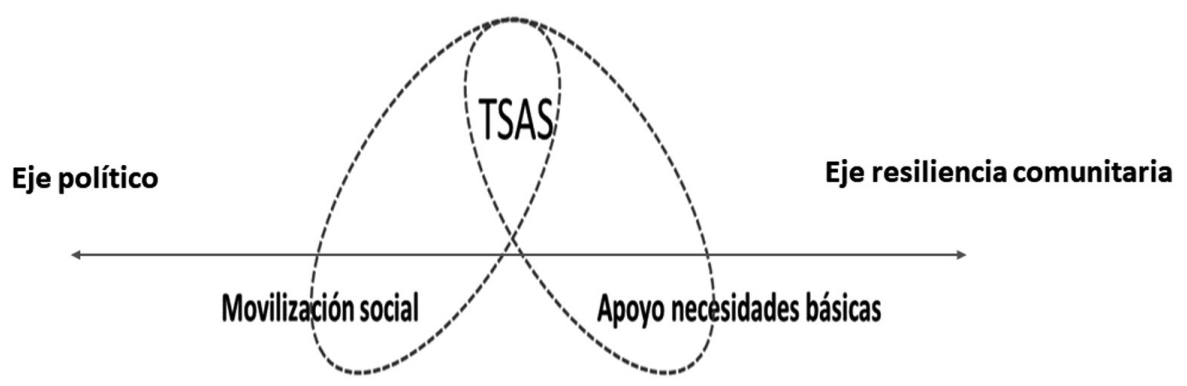

Fuente: Elaboración propia.

Los análisis realizados tras la Gran Recesión (Giudni y Grasso, 2018; Kousis, 2017), especialmente en algunos de los países del Sur de Europa (Italia, España y Grecia), muestran como la dimensión política de estas redes tuvieron una orientación más centrada en la protesta política en contraposición a países donde el impacto de la crisis fue menor (Alemania, Francia, Suecia, Reino Unido Suiza y Polonia). España, junto con Grecia, son los países con mayor actividad de protesta por parte de estos grupos. No significa que no promovieran otros tipos de actividades sino de la orientación principal. Sin duda, la apropiación en el discurso de la crisis económica y la condición social precaria de la ciudadanía potenció esta orientación (Zamponi y Bosi, 2018).

Un ejemplo típico de esta realidad en España fue la Plataforma de afectados por la Hipoteca (en adelante PAH). Este movimiento, caracterizado como red híbrida comunitaria y local (Alvarez de Andrés, Zapata y Zapata, 2015), es un caso paradigmático de orientación política, base comunitaria y gobernanza abierta. La PAH estuvo conformada desde los inicios por personas directamente afectadas además de otros grupos y personas de apoyo. Según Ferrán Giménez (2019) la subjetividad política de los activistas de la PAH estaba definida por la identidad de afectado y víctima. En algunos casos, esta orientación causó ciertos desencuentros con organizaciones del TSAS implicados en apoyos a la vivienda como prestación de servicios. Que la "protesta" fuese el eje destacado no significa que no existieran multitud de "propuestas" reveladoras, muchas de ellas proveniente del mundo de las economías alternativas. El Informe FOESSA (2014) en uno de sus capítulos (Zubero(Coord), 2014: 433-438) analizaba, bajo el epígrafe "las alternativas ya están aquí", un número muy relevante de prácticas sociales autogestionadas, colaborativas o alternativas. Ahora bien, la orientación fundamental presentaba una tendencia hacia la protesta y la politización. 
En el 2008 tuvo más político en sentido de denuncia, porque había un problema político.

(Entrevista 5)

Una parte del TS reivindican, es más políticos, más vinculados a su origen, más vinculado a la comunidad y más flexible en cuanto a determinados criterios... y viene haciendo propuestas concretas.

(Entrevista 4)

Sin embargo, en la pandemia han (re)emergido un conjunto de redes vecinales-comunitarias que, sin perder su dimensión política, se organizan en torno a la asistencia en las necesidades básicas.

Se hace incidencia política pero no partidista. Ya había otros que hacen esto, nosotros apoyamos a las vecinas (...) Ahora bien, tenemos líneas rojas y además de coordinarnos con la Administración, con los Servicios sociales, les pedimos que informaran de lo que se había hecho con cada familia que les derivábamos (...) Sí, somos un movimiento social que ha puesto en marcha a personas que nunca ha participado en estas iniciativas.

(Entrevista 2)

Aunque todavía los análisis están en fase preliminar se puede afirmar la importante presencia que están teniendo las redes vecinales en estos tiempos pandémicos. En la Comunidad de Madrid, cómo núcleo poblacional relevante, fueron atendidas más de 100.000 personas desde el primer confinamiento domiciliario, marzo del 2020, hasta mayo de 2021. Fueron 76 redes vecinales (de ellas 63 en la capital) las que, desde despensas comunitarias, aportaron ayuda en alimentos y productos de primera necesidad a unas 30.000 familias. Una labor gigantesca que fue desarrollada gracias al trabajo de más de 6.000 personas $^{8}$. También en Barcelona ${ }^{9}$, como otro núcleo poblacional importante, la presencia de las Xarxes de suport veïnal (Navarro 2021) están teniendo una importante labor.

Estas redes, especialmente en los momentos más duros del confinamiento, mostraron la capacidad de movilizar muchos recursos humanos, económicos y materiales en muy poco tiempo.

Empezamos de manera informal, con un grupo de wasap. Luego vino la web... todo hecho por vecinos (...) Fueron surgiendo grupos por distritos, las 5 despensas solidarias que funcionan de manera autónoma. Y así, hasta que algunas de estas iniciativas se han constituido legalmente para justificar donaciones. Han entrado más de 200.000 euros de donaciones particulares en muy poco tiempo, y por eso se crearon como asociaciones para tener un número de cuenta y para poder dar justificantes de donación. No es por aspiración, sino por necesidad.

(Entrevista 2)

8. Datos preliminares del estudio de la FRAVM sobre Redes vecinales-comunitarias. Recuperado de https://aavvmadrid.org/noticias/las-redes-vecinales-distribuyen-alimentos-a-mas-de-50-000-personas-en-toda-la-region/ 9. Se puede consultar https://suportpopular.org/y https://www.solivid.org/ para hacernos cargo de la magnitud. 
Además, el arraigo al territorio permite formar redes de carácter informal con una alta cualidad relacional. Esta relacionalidad produce "un patrimonio inmaterial que construye una necesaria riqueza no material" (Navarro, 2021).

Probablemente vaya a quedar poca cosa como iniciativa estructurada. Su respuesta es desde la inmediatez. Pero queda el buen recuerdo de que, la respuesta comunitaria, el apoyo mutuo y la autorganización son esenciales, y que habrá que fomentarlas y recurrir a ellas para resolver determinadas cuestiones. Una latencia que se podrá reactivar. Una demostración de cuál es el camino.

(Entrevista 5)

Al mismo tiempo, estas actividades netamente asistenciales (despensas comunitarias, apoyo higiene y ropa, etc.) adquieren una legitimidad transformadora. Si el TSAS se siente, en cierta medida, atrapado en el asistencialismo, las redes vecinales-comunitarias se despliegan con una amplia legitimidad social. Ésta le viene conferida por el trabajo de proximidad, entre pares, desde la ayuda mutua y con unos modelos de gobernanza abiertos.

La duda es si las instituciones del TSAS, forman parte del territorio, de las periferias. Si así fuera, la gente, te encuentra de forma espontánea cuando te necesita. Lo encuentras aún sin buscarlo. A los que ya estaban les han encontrado, y a los que no, no. Es cierto que hay entidades que formalmente son del TSAS y a las que se ha acudido, pero también a otras más pequeñas e informales que están en el territorio y que están en el imaginario de quienes las necesitan. La pregunta fundamental es si las ONG forman parte de las comunidades o no. Se trata del retorno a la comunidad. Y si las entidades no son parte, no se las encuentra.

(Entrevista 5)

Estas redes declaran que sus características esenciales son: agilidad, confianza, solidaridad, horizontalidad, apoyo mutuo, espontaneidad, independencia (Buj y Caso, 2021) y, afirman, que estas cualidades no destacan en un TSAS que se presenta profesionalizado, dependiente de los programas de las Administraciones públicas y burocratizado.

La diferencia entre estas iniciativas y las ONG es que no había horarios, ni un sistema de requisitos para acceder a sus alimentos... mucho más rápidos porque no tienen que pasar filtros.

(Entrevista 2)

Es importante destacar la amplía legitimidad de estas respuestas vecinales-comunitarias en los tiempos pandémicos ${ }^{10}$. Reconocimiento que ha llegado incluso a las instancias europeas otorgando el Premio ciudadano europeo 2020 a "Somos tribu"11 destacando el valor de la resiliencia comunitaria.

En este marco de emergencia de potencial comunitario, han cobrado visibilidad las Fundaciones comunitarias filantrópicas de procedencia americana, que están cobrando un desta-

10. Un trabajo que queda pendiente es el análisis de los titulares de prensa durante la Gran Recesión en los que el TSAS era presencia destacada y, sin embargo, durante la pandemia las Redes vecinales-comunitarias han tenido un mayor protagonismo y relevancia.

11. Recuperado de https://www.europarl.europa.eu/at-your-service/es/be-heard/prizes 
cado protagonismo. Son Fundaciones que promueven las capacidades comunitarias, locales y enraizadas en los territorios, como modelo básico de intervención. Más que el trabajo de sostenimiento de grandes organizaciones son programas de soporte de grupos comunitarios. Para la Fundación Carasso, una de las promotoras en España, de esta filantropía comunitaria, "el modelo de fundaciones comunitarias ha demostrado potenciar de manera decisiva aspectos tan cruciales como la profesionalización de la acción social, la correspondencia entre ésta y las necesidades de las comunidades, la concienciación y movilización para la acción de todo el conjunto de la sociedad y el vínculo social entre personas y entre organizaciones, en pro del bien común"12.

La Aga Khan Foundation USA y la Charles Stewart Mott Foundation definen la filantropía comunitaria como "personas de una misma localidad que se ayudan entre sí, compartiendo recursos para el bien común" con las siguientes características: "organizada y estructurada, autodirigida, de arquitectura abierta, que emerge de la sociedad civil y utiliza sus propios recursos y activos para construir una sociedad incluyente y equitativa" (Knight, 2012).

Estas Fundaciones, a través de una convocatoria de la Asociación Española de Fundaciones, ha apoyado muchas de las iniciativas vecinales mencionadas. Con imaginarios políticos diversos tanto las redes vecinales-comunitarias como las, menos reconocidas, Fundaciones comunitarias han mostrado la amplia legitimidad de las iniciativas que surgen de la comunidad para atender las necesidades más básicas de las personas desde la proximidad del territorio.

En este dinamismo que se produce (Gráfico 3) desde la Gran Recesión (movilización) a la crisis del coronavirus (apoyo necesidades básicas), el TSAS queda en una situación intermedia, centrado en las labores asistenciales, pero sin la dimensión política movilizadora activada durante la crisis financiera, y con el potencial comunitario erosionado durante la pandemia.

Formar parte de las comunidades es formar parte de los mismos procesos y verse afectados por los mismos problemas.

(Entrevista 5)

El TSAS ha empezado a buscar espacios de crecimiento, más ligados a la intervención social que a la incidencia y a la transformación política. No tanto por miedo a perder fondos como castigo a la denuncia, sino para conseguir la máxima financiación posible para poder hacer más cosas, llegando incluso a reorientan su acción, en función de que haya financiación.

(Entrevista 3)

En el III Plan Estratégico de TSAS en España se destacaba que existía "un proceso larvado de reducción de la base social del TSAS y de una cierta desmovilización" (POAS, 2016:10). Es decir, el eje movilizador no presentaba una energía relevante, y por otra parte la base social, eje de la resiliencia comunitaria, estaba reducida. Veámoslo con más detenimiento.

Con respecto al eje movilizador, observamos que tras el movimiento de los indignados en 2011 se construyeron grandes recelos, entre el TSAS y los movimientos sociales, que llegan hasta nuestros días. Esta dinámica entre ambas realidades sociales es ambigua y paradójica, de atracción y repulsión. La crítica por la ausencia del TSAS en los movimientos de protesta y por el asistencialismo apolítico del TSAS, manifestó de manera singular la bifurcación de

12. Recuperado de https://www.fondationcarasso.org/es/filantropia/fundaciones-comunitarias/ 
prácticas, estilos y pertenencias, que venían gestándose desde años atrás (Mora, 2001; Zubero, 2018). Aunque hay relaciones sinérgicas y "simbiosis clandestinas" entre el TSAS y los movimientos sociales (EAPN-ES, 2019) y exista una cierta hibridación entre ellos (Della Porta, 2020), siguen existiendo diferencias notables entre ambas dimensiones de la acción colectiva (EAPN-ES, 2019). Además de diferencias en la base social entre ellos (Zubero, 2018) y la formalidad organizativa del TSAS no permita una participación más horizontal y participativa como los movimientos sociales (Kousis y Paschou, 2017) la gran diferencia está en la orientación política más marcada por los movimientos sociales.

Nuestro trabajo (el del TS) debería ser generar espacios de protagonismo y participación.

(Entrevista 6)

Lo que debería definir al TS es la participación, pero te encuentras iniciativas que no tienen una base participativa de entrada.

(Entrevista 3)

Respecto a las iniciativas vecinales: han estado muy vinculada a movimientos con ideología política, que hicieron redes que no diferenciaban tanto entre el que ayuda y el que es ayudado... esto sí pueden favorecer que se consoliden como experiencias más comunitarias, con reflexión... luchando juntos por los derechos...

(Entrevista 4)

Como el mismo TSAS reconocía, "en los años de crisis (2009-2014) han emergido nuevas respuestas de la ciudadanía ante situaciones de incertidumbre, aumento de las desigualdades y relativa deslegitimación de algunas instancias y actores del ámbito público. Estos nuevos movimientos sociales han logrado nuevas maneras de incidencia y presencia política e institucional, visibilidad y apoyo popular en su dimensión de protesta y denuncia, en un periodo convulso en el que el TSAS ha estado en general más enfocado a la atención de urgencias sociales, por una parte, y a sus propios ajustes para mantener su posición en relación con sus financiadores" (POAS, 2017: 23).

Con respecto al eje de resiliencia comunitaria, observamos como el TSAS tiene "pérdida de capacidad para crear tejido social al mismo tiempo que, relativamente, se ha debilitado la capacidad reivindicativa y defensa de los derechos sociales del Tercer Sector de Acción Social, frente a una mayor concentración en la prestación de servicios" (POAS, 2016:10).

Lo que diferencia al TSAS es en realidad el nivel de profesionalización, frente a otras iniciativas que puede que algún día se conviertan en TS. Y que están desarrollando acción social. Son espacios especializados y técnicos, pero con una cierta carencia de base social, aunque haya socios económicos. Hay financiadores, claro, pero a veces les falta el respaldo de la sociedad civil.

(Entrevista 3)

De hecho, esta pérdida de relevancia comunitaria es causa del descenso de confianza y legitimidad del TSAS. Germán Jaráiz y Rosalía Mota destacaban que la retirada de los espacios de participación asociativa de proximidad y de la lógica vecinal-comunitaria (Jaraíz y Mota, 2019) eran aspectos esenciales en la desconfianza hacía el sector. Como hemos visto en la pandemia la lógica comunitaria legitima, visibiliza y empodera a las comunidades. 


\section{Conclusiones}

Tal y como hemos venido señalando, el TSAS está evolucionando hacia formas organizativas híbridas, características de un modelo abierto que acoge culturas organizativas diversas y estructuras flexibles. En este proceso de hibridación distinguimos cuatro campos intermediarios esenciales: la economía social, el emprendimiento social, los movimientos sociales y las nuevas formas de resiliencia comunitaria. Afianzar estos campos de manera productiva e innovadora será un eje fundamental en el despliegue del TSAS en los próximos años.

La realidad social en los últimos 10 años, atravesada por dos profundas crisis, ha impactado en el TSAS (en su despliegue de acciones, e incluso en sus funciones y sentido), que ha tratado de dar respuesta al incremento intenso de las necesidades básicas de parte de su población.

El TSAS en la Gran Recesión se construyó en tensión con las movilizaciones sociales emergentes. Estas tuvieron un claro perfil político frente a la fisonomía asistencial del TSAS. Este perfil ha sido compartido con los países del Sur de Europa. Sin embargo, durante la crisis por Covid-19, el TSAS ha mantenido su perfil asistencial compartiendo ese espacio con las Redes vecinales comunitarias constituidas con una base más social. Esta resiliencia comunitaria, según muestran los primeros estudios, parece que ha sido común en los países de nuestro entorno. En este sentido el TSAS vive con tensión su falta de proximidad y base social, tal como han mostrado los estudios desde hace años en España.

Esto hace vislumbrar retos que, en función de cómo se aborden, podrán tener efectos tanto en su orientación, como en el desarrollo e incluso en la organización del mismo:

- En relación al rol asistencial, el TSAS necesita encontrar formas alternativas que no menoscaben la dignidad de las personas que solicitan su ayuda.

- Además, el TSAS deberá desplegar su capacidad de implicar a otros agentes (donantes, empresas, Administración pública...) en los procesos de reflexión y en la búsqueda de soluciones. Esto conllevará procesos "educativos" acordes a las orientaciones y objetivos perseguidos por el TSAS (p.e. entrega de tarjetas monedero vs entrega de bolsas de alimentos).

- El TSAS debe desarrollar su capacidad innovadora que, a diferencia de la Administración pública (mucho más limitada) le permitirá implementar acciones en distintos ámbitos y en relación directa con la sociedad civil y con las redes vecinales-comunitarias.

- Pero, además, es necesario que el TSAS no pierda su dimensión más política (en un sentido amplio del término) y que profundice en las raíces y las causas de las problemáticas que atiende.

- Esta dimensión afecta a su relación con la Administración pública en dos aspectos, el primero, en cuanto a la incidencia política (e incluso la denuncia) dirigida a quien financia parte de su actividad; y el segundo, porque la búsqueda de recursos para este tipo de tareas suele requerir un mayor esfuerzo para las entidades.

Por último, cabe destacar que la presencia cotidiana en los diversos territorios es un elemento clave en la configuración de sus relaciones con otros protagonistas. Parte de su reconoci- 
miento y de sus alianzas potenciales dependerá de esta presencia más allá de los momentos de crisis. Si uno de los objetivos fundamentales del TSAS es fomentar la participación de quienes habitualmente quedan excluidos de diversos ámbitos (sociales, económicos, políticas, culturales...), su arraigo y vinculación con la comunidad (usuarios, donantes, voluntarios, empresas, Administración pública...) serán aspectos definitivos para su consecución. Para ello, el TSAS tendrá que abrirse a formas más abiertas de gobernanza, promoviendo el empoderamiento de las personas y comunidades afectadas y rompiendo el gap entre profesionales y participantes.

Contribución de cada autor/a: Sebastián Mora Rosado: aportación teórica, revisión bibliográfica, elaboración y análisis de entrevistas; Francisco Javier de Lorenzo Gilsanz: aportación teórica, revisión bibliográfica, elaboración y análisis de entrevistas.

\section{Bibliografía}

ÁLVAREZ DE ANDRÉS, E., ZAPATA, M.J. \& ZAPATA, P. (2015): "Stop the evictions! The diffusion of networked social movements and the emergence of a hybrid space: The case of the Spanish Mortgage Victims Group", Habitat International, 46, 252-259.

DOI:10.1016/j.habitatint.2014.10.002.

BATTHYÁNY, K. \& CABRERA, M. (2011): Metodología de la investigación en Ciencias Sociales, Montevideo: Universidad de la república de Uruguay.

BISHOP, M. \& GREEN, M. (2009): Philanthrocapitalism. How the Rich Can Save the World, New York: Bloomsbury Press.

BUJ, C. \& CASO, A. (2021): Manual práctico para el desarrollo de redes comunitarias, Zaragoza: Proyecto Contra viento y Marea-European cultural Foundation.

CAMERON, S. (2021): "Civic engagement in times of economic crisis: a cross-national comparative study of voluntary association membership", European Political Science Review, 13, 265283. DOI:10.1017/S1755773921000060

CÁRITAS (2021): Un año acumulando crisis. La realidad de las familias acompañadas por Cáritas en enero de 2021, Madrid: CÁRITAS.

CHAVES, R., MONZÓN, J.L. \& ZARAGOZA, G. (2013): “La economía social: concepto, macromagnitudes y yacimiento de empleo para el trabajo social", Cuadernos de Trabajo Social, 26(1), 19-29. DOI:https://doi.org/10.5209/rev_CUTS.2013.v26.n1.40269

CRUZ ROJA (2021): El impacto de la COVID-19 en la población atendida por Cruz Roja a través del Plan RESPONDE, Madrid: Cruz Roja. 
CHAVES, R. \& ZIMMER, A. (2017): El Tercer Sector en España y Europa. Crisis y resiliencia, Valencia: Fundación General de la Universidad de Valencia.

CONDE, F. (2019): "Apuntes sobre el análisis y la interpretación de los "emergentes discursivos" en el análisis sociológico de los discursos", Encrucijadas. Revista de Ciencias Sociales, 17, v1701.

DELLA PORTA, D. (2020): "Building Bridges: Social Movements and Civil Society in Times of Crisis". Voluntas: International Journal of Voluntary \& Nonprofit Organizations, 31(5), 938-948. D0I:https://doi.org/10.1007/s11266-020-00199-5

EAPN-ES (2019): Tercer Sector de Acción Social, Movilización Social y Voluntariado ¿Transformando juntos?, Madrid: EAPN-ES y PVE.

ESTIVIL, J. (2015): “El asociacionismo vector de la economía solidaria”. In: J.L. Laville (Ed.), Asociarse para el bien común. Tercer Sector, Economía social y Economía solidaria, Barcelona: Icaria, p. 216.

EUROFUND (2020): Living, working and COVID-19, COVID-19 series, Luxemburg: Publications Office of the European Union.

EVERS, A. \& EWERT, B. (2015): "Social innovation for social cohesion". In: A. Nicholls, J. Simon \& M. Gabriel (Eds.), New frontiers in social innovation research, London: Palgrave Macmillan, 107-127.

FANTOVA, F. (2019): "Los agentes en la transformación de los servicios sociales", Cuadernos Trabajo social, 32(2), 431-444.

FOESSA (2014): VII Informe sobre exclusión y desarrollo social en España, Madrid: FOESSA.

FOESSA (2019): VIII Informe. Sobre la exclusión y el desarrollo social en España, Madrid: FOESSA.

GARCÍA ROCA, J. (2012): Reinvención de la exclusión en tiempos de crisis, Madrid: Cáritas-F0ESSA.

GARCÍA, G., BARRIGA, L., RAMÍREZ, J.M., ZUBIRÍA, A., VELASCO, L. \& IZQUIERDO, A. (2017): Índice DEC, Madrid: Asociación Estatal de Directoras y gerentes de Servicios Sociales.

GENTILINI, U. (2013): Banking on Food: The State of Food Banks in High-income Countries, Working Paper, University of Sussex, Institute of Development Studies, 415, 1-18.

D0I:10.1111/j.2040-0209.2013.00415.x

GIUDNI, M. \& GRASSO, M. (2018): "Alternative Action Organizations: Social Solidarity or Political Advocacy?", American Behavioral Scientist, 62(6), 778-795.

D0I:https://doi.org/10.1177/0002764218768855 
GÓMEZ, M. (Ed.) (2020): El Tercer Sector de Acción Social en España 2019. Nuevos horizontes para un nuevo contexto socio-político, Madrid: POAS.

IBARRA, P. \& TEJERINA, B. (1998): "Hacia unas nuevas formas de acción colectiva". In: P. Ibarra y B. Tejerina (Eds.), Los movimientos sociales. Transformaciones políticas y cambio cultural, Madrid: Trotta, 9-22.

JARAÍZ, G. \& MOTA, R. (2019): Capital Social y Cultural en España. Análisis de tendencias y transformaciones en el periodo 2013-2018, Madrid: FOESSA.

KNIGHT, B. (2012): The Value of Community Philanthropy - Results of a Consultation, Washington D.C: Aga Khan Foundation USA-Charles Stewart Mott Foundation.

KOUSIS, M. (2017): "Alternative forms of resilience confronting hard economic times: A South European Perspective", Partecipazione E Conflitto, 10(1), 119-135.

D0I:10.1285/i20356609v10i1p119

KOUSIS, M. \& PASCHOU, M. (2017): "Alternative forms of resilience: a typology of approaches for the study of citizen collective responses in hard economic times", Partecipazione E Conflitto, 10(1), 136-168. D0I:10.1285/i20356609v10i1p135

MAIER, F., MEYER, M. \& STEINBEREITHNER, M. (2016): "Nonprofit Organizations Becoming Business-Like: A Systematic Review", Nonprofit and Voluntary Sector Quarterly, 45(1), 64-86. DOI:10.1177/0899764014561796

MEIJS, L., HANDY, F., SIMONS, FJ. \& ROZA, L. (2019): "A Social Innovation: Addressing Relative Food Insecurity and Social Exclusion", Voluntas: International Journal of Voluntary \& Nonprofit Organizations, 31(5), 1-13. DOI:10.1007/s11266-019-00105-8

MINISTERIO DE SANIDAD (2020): Redes comunitarias en la crisis de COVID19, Madrid: mscsb.

MONZÓN, J.L \& CHAVES, R. (2017): Evolución reciente de la economía social en la Unión Europea, Bruselas: Comité Económico y Social Europeo (CESE) y CIRIEC International.

MORA, S. (2001): “¿Es el voluntariado un movimiento social?”. In; A. García \& J.M. Martínez (Eds.), Ciudadanía, voluntariado y participación, Madrid: Dykinson, 79-90.

MORA, S. (2020): "Pandemia social: exclusión, desigualdad y discriminación en tiempos de COVID-19". In: R. Amo \& F. de Montalvo (Eds.), La humanidad puesta a prueba. Bioética y COVID-19, Madrid: Universidad Pontificia Comillas, 360-373.

MOULAERT, F. \& AILENEI, O. (2005): "Social Economy, Third Sector and Solidarity Relations: A Conceptual Synthesis from History to Present", Urban Studies, 42(11), 2037-2053. DOI:https://doi.org/10.1080/00420980500279794 
MOULAERT, F., MARTINELLI, F., SWYNGEDOUW, E. \& GONZÁLEZ, S. (2005): “Towards Alternative Model(s) of Local Innovation", Urban Studies, 42(11), 1960-1990.

DOI: https://doi.org/10.1080/00420980500279893

NAVARRO, M. (2021): "Redes vecinales de apoyo y estrategias de afrontamiento en familias empobrecidas en Barcelona en tiempos de pandemia", Gazeta de Antropología, 37(1), artículo 2. DOI:10.30827/Digibug.69640

PAARLBERG, L.E., LEPERE-SCHLOOP, M., WALK, M., AI, J. \& MING, Y. (2020): "Activating Community Resilience: The Emergence of COVID-19 Funds Across the United States", Nonprofit and Voluntary Sector Quarterly, 49(6), 1119-1128. D0I:10.1177/0899764020968155

PAHL, B. \& ZIMMER, A. (2017): "Obstáculos al desarrollo del Tercer Sector en Europa. Modelos y estrategias de resiliencia”. In: R. Chaves-Ávila \& A. Zimmer (coord.) (Eds.), El Tercer Sector en España y Europa Crisis y resiliencia, Valencia: Universitat de València, 131-153.

PAPE, U., CHAVES, R., PAHL, B., PETRELLA, F., PIELINSKI, B. \& SAVALL-MORERA, T. (2017): "Trabajando bajo presión en Europa: Crisis económica y desarrollo del Tercer Sector en Europa". In: R. Chaves \& A. Zimmer (Eds.), El Tercer Sector en España y Europa. Crisis y resiliencia, Valencia: Fundación General de la Universidad de Valencia, 155-179.

PENG, X. \& LIANG, C. (2019): "Before Nonprofit Organisations Become Social Enterprises", Voluntas: International Journal of Voluntary \& Nonprofit Organizations, 30(3), 460-474.

DOI:https://doi.org/10.1007/s11266-018-00071-7

PÉREZ DE ARMIÑO, K. (2014): "Erosion of rights, uncritical solidarity and food banks in Spain". In: G. Riches \& T. Silvasti (Eds.), First World Hunger Revisited. Food Charity of the Right to Food?, Basingstoke (UK) and New York: Palgrave Macmillan, 131-145.

PLATAFORMA DEL TERCER SECTOR (2011): Documento base del Proyecto de articulación del Tercer Sector en España, Madrid: PTS.

POAS (2015): El Tercer Sector de Acción Social en 2015: impacto de la crisis, Madrid: POAS.

POAS (2016): Diagnóstico y cuestiones para la reflexión estratégica III Plan Estratégico, Madrid: Plataforma de ONG de Acción Social.

POAS (2017): III Plan Estratégico del Tercer Sector de Acción Social 2017-2021, Madrid: Plataforma de ONG de Acción Social.

RODRÍGUEZ CABRERO, G. \& MARBÁN, V. (2015): Análisis prospectivo sobre los retos actuales y futuros del TSAS. El tercer Sector en la Unión Europea. Situación actual y tendencias (parte 1), Madrid: POAS. 
RUIZ, R. (2016): “El Tercer Sector de Acción Social en 2015: impacto de la crisis", Revista Española Del Tercer Sector, 34, 217-227.

SANZ, J. (2019): "Economía social y solidaria, emprendimiento social y economía popular en la sociedad post-crisis", Revista De Antropología Social, 28(2), 205-226.

D0I:https://doi.org/10.5209/raso.65612

STANZANI, S. (2005): "Terzo settore e differenziacione sociale: una teoria relazionale", In: P. Donati \& P. Terenzi (Eds.), Invito alla sociologia relazionale. Teoria e applicazioni, Milano: Franco Angeli, 198-216.

VALENZUELA-GARCÍA, H., MOLINA, J.L., LUBBERS, M.J., ESCRIBANO, P. \& FUENTES, S. (2019): "Emprendimiento Social. Autoempleo y extracción del valor en la era post-crisis", Revista de Antropología Social, 28(2), 371-390. DOI:https://doi.org/10.5209/raso.65619

VALLÉS, M. (1999): Técnicas cualitativas de investigación social, Madrid: Síntesis Ed.

VIDAL, F. (2021): Cuando el mundo paró. Diario del coronavirus, Madrid: PPC.

WRIGHT, E.0. (2014): Construyendo utopías reales, Madrid: Akal.

ZAMPONI, L. \& BOSI, L. (2018): "Politicizing Solidarity in Times of Crisis: The Politics of Alternative Action Organizations in Greece, Italy, and Spain". American Behavioral Scientist, 62(6), 796-815. DOI:https://doi.org/10.1177/0002764218768861

ZUBERO, I. (2015): “La relación entre el TSAS, los movimientos sociales y las nuevas formas de participación en la sociedad". In: G. Rodríguez-Cabrero \& V. Marbán (Eds.), Análisis prospectivo sobre los retos actuales y futuros del TSAS (parte 2), Madrid: P0AS, 64-100.

ZUBERO, I. (2018): "El Tercer Sector como movimiento voluntariadista: una propuesta para repensar la identidad del TSAS desde el paradigma de la democracia del cuidado", Revista Española del Tercer Sector, 38, 43-68.

ZUBERO, I. (Coord.) (2014): “QQué sociedad saldrá de la actual crisis? ¿Qué salida de la crisis impulsará esta sociedad?”. In: FOESSA (Ed.), VII Informe sobre exclusión y desarrollo social en España, Madrid: FOESSA, 397-449. 


\section{Anexo}

Se han realizado 7 entrevistas en profundidad realizadas a personas que ocupan diversas posiciones en el ámbito social. Dichas entrevistas han buscado una cierta diversidad territorial ( 3 en ciudades de entre 50.000 y 350.000 habitantes y otras 4 en ciudades de tamaño grande de más de 500.000 habitantes).

- Entrevista 1: Coordinadora de Acción social de entidad del TSAS, Albacete.

- Entrevista 2: Fundadora de red vecinal creada durante la pandemia, Madrid.

- Entrevista 3: Miembro de la Junta del Colegio de Trabajo social y Responsable de programa social en entidad del TSAS, Málaga

- Entrevista 4: Trabajadora social en entidad de TSAS, Barcelona.

- Entrevista 5: Miembro Asociación de Vecinos y experto en TSAS, Madrid

- Entrevista 6: Gerente de entidad de TSAS, Segovia.

- Entrevista 7: Responsable de programa social en entidad del TSAS, Salamanca. 